\title{
Using False Colors to Protect Visual Privacy of Sensitive Content
}

\author{
Serdar Çiftçi ${ }^{a}$, Pavel Korshunov ${ }^{b}$, Ahmet Oğuz Akyüz ${ }^{a}$, and Touradj Ebrahimi ${ }^{b}$ \\ ${ }^{a}$ Department of Computer Engineering, Middle East Technical University, Ankara, Turkey; \\ ${ }^{b}$ Multimedia Signal Processing Group, EPFL, Lausanne, Switzerland
}

\begin{abstract}
Many privacy protection tools have been proposed for preserving privacy. Tools for protection of visual privacy available today lack either all or some of the important properties that are expected from such tools. Therefore, in this paper, we propose a simple yet effective method for privacy protection based on false color visualization, which maps color palette of an image into a different color palette, possibly after a compressive point transformation of the original pixel data, distorting the details of the original image. This method does not require any prior face detection or other sensitive regions detection and, hence, unlike typical privacy protection methods, it is less sensitive to inaccurate computer vision algorithms. It is also secure as the look-up tables can be encrypted, reversible as table look-ups can be inverted, flexible as it is independent of format or encoding, adjustable as the final result can be computed by interpolating the false color image with the original using different degrees of interpolation, less distracting as it does not create visually unpleasant artifacts, and selective as it preserves better semantic structure of the input. Four different color scales and four different compression functions, one which the proposed method relies, are evaluated via objective (three face recognition algorithms) and subjective (50 human subjects in an online-based study) assessments using faces from FERET public dataset. The evaluations demonstrate that DEF and RBS color scales lead to the strongest privacy protection, while compression functions add little to the strength of privacy protection. Statistical analysis also shows that recognition algorithms and human subjects perceive the proposed protection similarly.
\end{abstract}

Keywords: Visual privacy protection, false color visualization, objective evaluation, subjective assessment.

\section{INTRODUCTION}

The advances in imaging technologies, widespread use of social networks, and rapid adoption of surveillance systems have created a situation where we are under the constant surveillance with daily violation of personal privacy. While social networks, photo and video sharing platforms, and cloud based services provide privacy and security protection mechanisms (albeit being rudimentary and inefficient as recent privacy scandals demonstrated), little is done for privacy protection in video surveillance systems.

One reason for the lack of use of privacy protection tools in video surveillance is the problem of balancing between privacy, the amount of personal information visible in a video, and intelligibility, the amount of visible information that is necessary to perform a surveillance task. An ideal video surveillance system should protect privacy without sacrificing intelligibility. This means, for instance, that unauthorized individuals should not be able to recognize people in a protected surveillance video but, if need be, authorities such as police, should be able to access the full content of the video during a potential criminal investigation. Furthermore, it should be possible to infer information from the protected video that is useful for the surveillance application without revealing the identities of the people. For example, determining the crowd density in a given region, the direction in which a group of people is moving, or the actions performed by people should be possible without revealing the identities of each individual involved in these scenarios.

Although many methods for privacy protection exist, most of them rely on computer vision algorithms, such as face or person detection, for identifying the privacy sensitive regions where the protection should be applied to. However, computer vision algorithms are not always accurate and may fail in certain cases such as poor capture conditions, noise in the captured

Further author information: (Send correspondence to Serdar Çiftçi)

Serdar Çiftçi: sciftci@ ceng.metu.edu.tr,

Pavel Korshunov: pavel.korshunov@epfl.ch,

Ahmet Oğuz Akyüz: akyuz@ ceng.metu.edu.tr,

Touradj Ebrahimi: touradj.ebrahimi@epfl.ch 
frames, partial occlusion of the individuals, and non-ideal camera viewpoints or field-of-views. The failure even in a single frame of a video can lead to the loss of the privacy protection efforts. Therefore, a method that is independent on the underlying computer vision algorithms is needed for more robust privacy protection.

This paper proposes a computer vision independent method that utilizes simple intensity compression/expansion schemes and false color visualizations. To this end, the intensity values of a face image are first compressed and then transformed to a different color scale. For compression, three different functions are used, including logarithmic, sigmoidal, and histogram equalization. An uncompressed condition is also used to evaluate the effectiveness of only using false colors. For false coloring, four different palettes are tested, namely the rainbow, Radiance default ${ }^{*}$, heated-body, and the linearized optimal color scales. The objective (based on the evaluation framework proposed $\mathrm{in}^{2}$ ) and crowd-based subjective (based on the approach by Rogowitz and Kalvin ${ }^{3}$ ) experiments, were conducted using 100 images of the public FERET face dataset ${ }^{4}$ and show that the proposed approach can effectively protect privacy of faces. Furthermore, the proposed scheme is reversible in a way that all operations can be reverted to obtain the original images. Finally, the protected images retain most of the information necessary for the surveillance task without revealing personal identifiable details.

Therefore, the following are the main contributions of the paper:

- Privacy protection method based on false color scale and intensity compression is proposed. Because the method preserves the semantic structure of an image, it can be applied to the whole image without the need of identifying privacy sensitive regions using typically inaccurate computer vision algorithms.

- Both objective and subjective evaluations of the method are performed, and their results are compared. Four color scales and four compression schemes are evaluated.

- The detailed analysis is performed to determine which scale is the most suitable for privacy protection in cases when both machines and humans are the observers.

\section{BACKGROUND AND RELATED WORK}

A large number of privacy protection methods are proposed in the literature which can be classified into two major groups. The first group of algorithms determine a region of interest (ROI) from the input frame and applies privacy protection only in this region. In the second group, privacy protection is performed on the entire frame. In the remainder of the paper we refer to these two groups as local and global methods respectively. Most of the existing privacy protection methods fall into the local category. The chief drawback of local methods is their reliance on computer vision techniques. That is, if the sensitive regions are not correctly identified, the privacy of the recorded individuals may be compromised.

Privacy protection algorithms can be classified along other dimensions as well. For example, some privacy methods are format/compression dependent while others are format/compression independent. The former methods only work for certain image or video formats such as JPEG or MPEG. Format independent methods do not place any restriction on the format of the content that they process. Privacy protection methods can also be classified based on their reversibility. Reversible methods enable the original image or video to be recovered from the protected versions assuming that a secret key is known. Irreversible methods, on the other hand, do not provide a mechanism to obtain the originals.

The three simplest methods of privacy protection are masking, blurring, and pixelation. Masking corresponds to painting the sensitive regions with an opaque color (e.g., insertion of a black box). Although this maximizes privacy, it is not only irreversible but it may also prevent acquiring non-sensitive information as well. Blurring involves smoothing the sensitive regions with a blur kernel. Using a large kernel radius may enhance privacy protection but it may also hinder reversibility. Using small kernels, on the other hand, may not ensure sufficient privacy. Pixelation methods transform the selected regions into a mosaic-like pattern effectively reducing the resolution of the sensitive regions. Their advantages and disadvantages are similar to that of blurring.

More advanced privacy protection methods have also been proposed such as warping. ${ }^{5}$ In warping, a set of key points are determined by using face detection techniques. These key points' coordinates are shifted according to a warping strength parameter and the new intensity values are determined by using interpolation. Warping is local and compression independent. Its reversibility depends on the strength of the warping applied. It has been shown that while low warping

${ }^{*}$ This is the default color scale used to visualize radiance maps in the Radiance global illumination software. ${ }^{1}$ 
strength values make the method reversible it may not provide sufficient protection against both human observers and face recognition algorithms. Using high values, on the other hand, may render the warped images irreversible. Furthermore, high warping values often result in visually disturbing face rendering.

Another related privacy protection approach is called morphing. ${ }^{6}$ In morphing, the goal is to find an average face image between the source and the target faces according to a given interpolation level. The source face corresponds to the face of the individual whose identity must be preserved. The target face is any generic human face. The method first divides both images into Delaunay triangles ${ }^{7}$ and transforms the vertices of the source image toward the vertices of the target image. The pixel intensities are also interpolated with respect to a second parameter. Morphing is compression and format independent. It is also reversible unless the source image is morphed perfectly to the target image and the target image is known. Its security can be ensured by encrypting the key points and randomizing the interpolation level and the pixel interpolation values for each triangle. However, as the algorithm begins with triangulating the face images, it may fail to work in cases where the faces are not captured from ideal angles.

Region based scrambling is another technique to protect privacy in video surveillance. ${ }^{8}$ First, the region to be scrambled (ROI) is estimated. Next, the signs of the AC and DC coefficients of discrete cosine transform (DCT) are pseudorandomly inverted. For security and reversibility, the seed value of the pseudorandom number generator is encrypted. Although scrambling ensures that the protected region is unrecognizable (as it appears as random noise), it also prevents acquiring non-sensitive information from the scrambled region. Furthermore, the method is format/compression dependent.

Privacy protection can also be accomplished by removing the sensitive parts of the frames of a video. The removed parts create holes in the resulting frames. These holes can be filled with image in-painting techniques. ${ }^{9}$ If the background of the frame is static then these holes can be filled with the information from the other frames. Otherwise, if the background is dynamic, the holes can be filled by using the information from the neighboring pixels.

Encrypting visual objects, such as shapes and textures in an image content that is partitioned in hierarchical trees can be performed with a method called Secure Shape and Texture SPIHT (SecST-SPIHT where SPIHT is an abbreviation for Set Partitioning in Hierarchical Trees). ${ }^{10}$ SecST-SPIHT encrypts shapes and textures and ensures that reconstruction is not possible without knowing the decryption key. It is reversible but it does not permit non-private information to be extracted from a video as the output does not contain any meaningful visual information.

A recent work by Erdélyi et al., called adaptive cartooning, converts an image into an abstracted cartoon-like version. ${ }^{11}$ The algorithm's main steps are smoothing and edge enhancement. The areas with similar color values are smoothed and the areas with color discontinuities (edges) are accentuated. This method can be applied to an ROI or the whole image. Thus it can be classified as both a local and global method. However, it is irreversible.

\section{FALSE COLOR BASED PRIVACY PROTECTION}

The core of the proposed method involves representing images in a different color scale to distort private information while preserving intelligibility. The rationale for this is based on the fact that the human visual system is particularly tuned to recognize faces when seen under standard illumination. If this illumination changes, for example by moving the light source such that it illuminates a face from the bottom rather than the top, it becomes difficult to recognize even familiar faces. Furthermore, earlier research suggests that if faces are represented in nonmonotonic color scales, it becomes much harder for people to recognize them. ${ }^{3}$

Based on these ideas, we first transform an image (containing faces) using a point-wise compression or expansion function. The purpose of this step is to bring together or spread apart the intensity distribution of the pixel values. We then transform the resulting image into a different color scale. In the following subsections, both the compression/expansion algorithms and the color scales are explained in more details.

\subsection{Compression/Expansion Stage}

The purpose of compression/expansion stage is to induce a change in the intensity distribution of an input image. We have experimented with logarithmic and sigmoidal functions as compressive transformations and histogram equalization as an expansion transformation. During the experiments, we have also tested a no-transformation case (abbreviated by NOP) 
to understand whether this initial step has a significant influence on the results. All of these operations are applied on the intensity image which is computed from an RGB image as follows ${ }^{\dagger}$ :

$$
Y=0.216 R+0.7152 G+0.0722 B
$$

Logarithmic scaling (LOG): Logarithmic scaling scales the logarithm values of the intensity image to the $[0,1]$ range:

$$
f_{\log }(Y)=\frac{\log (Y+\epsilon)-\log \left(Y_{\min }+\epsilon\right)}{\log \left(Y_{\max }+\epsilon\right)-\log \left(Y_{\min }+\epsilon\right)}
$$

Here, a small value $\left(\epsilon=10^{-6}\right)$ is added to intensity values to avoid singularity for black pixels. ${ }^{12}$

Sigmoidal compression (SIG): This compression technique, inspired from the photographic tone mapping operator, ${ }^{13}$ compresses the intensity values through an S-shape function applied after an initial intensity scaling:

$$
f_{\text {sig }}(Y)=\frac{\alpha Y / \bar{Y}}{1+\alpha Y / \bar{Y}}
$$

where $\alpha$ denotes a user-defined key value and $\bar{Y}$ is the log-average intensity value computed as:

$$
\bar{Y}=\exp \left(\frac{1}{N} \sum_{x, y} \log (Y(x, y)+\epsilon)\right) .
$$

For the current experiments, $\alpha=0.18$ and $\epsilon=10^{-6}$ values are used. For both logarithmic and sigmoidal scaling the color mapping algorithm is defined in Algorithm 1, where one needs to substitute $f_{l o g}$ or $f_{\text {sig }}$ for $f$. In this algorithm, $\mathbf{C}_{\mathbf{m}, \mathbf{n}}$ is the false color value found in the given palette:

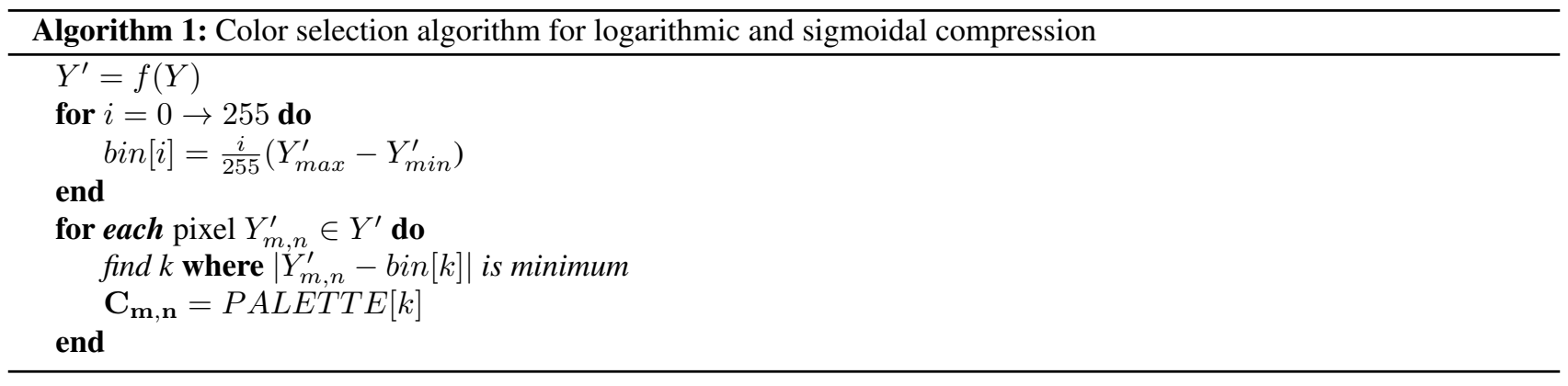

Histogram Equalization (HIS): Histogram equalization redistributes the intensity values such that each bin contains equal number of pixels. ${ }^{14}$ Instead of computing histograms, bin boundaries and palette indices based on the distance of the luminance values to these boundaries are directly computed as shown in Algorithm 2.

\subsection{Color Scale Selection}

Following the intensity compression stage, the colors of an image are then scaled according to one of the four color scales presented in Figure 1 and summarized below:

Rainbow scale (RBS): RBS is also called the spectral scale since the ordering of the colors is roughly based on their wavelength. The palette of this scale is generally produced by varying the hue attribute in a color space such as HSV and keeping the other attributes constant. RBS has a nonmonotonic perceived intensity progression.

\footnotetext{
${ }^{\dagger}$ We do not use the term luminance here as the input images are in a non-linear and uncalibrated color space.
} 

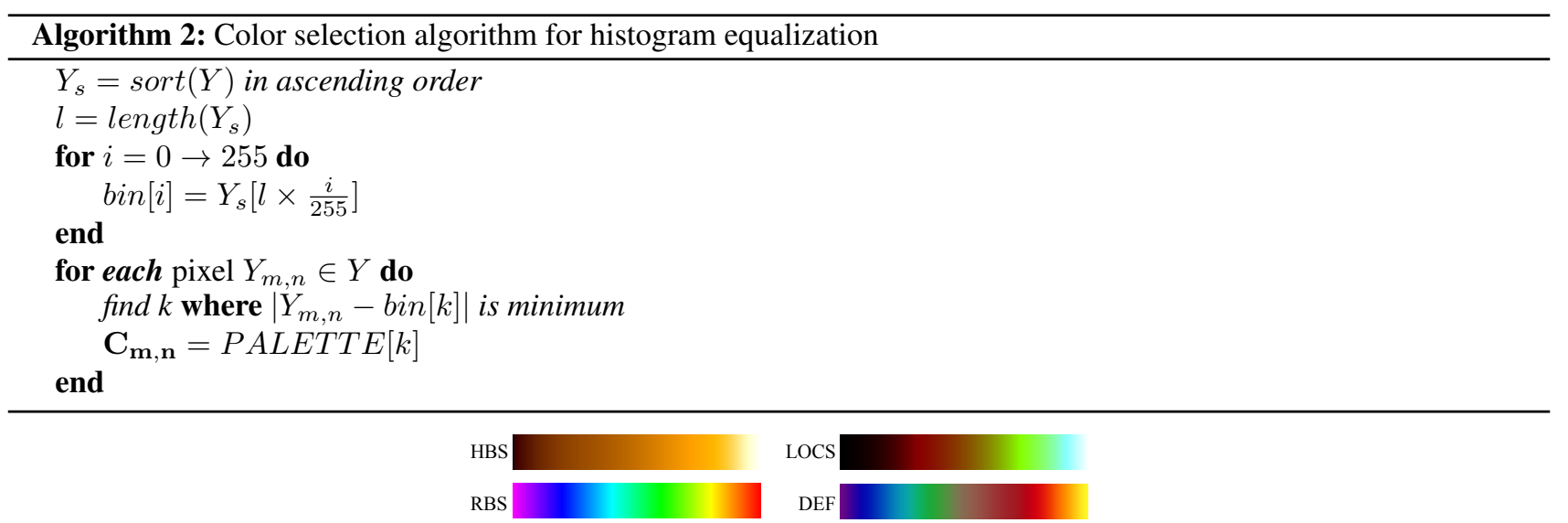

Figure 1: Color scales used in this study (see Appendix for numerical values).

Heated-body scale (HBS): In HBS, colors progress from black to white while passing through orange and yellow. The advantage of this scale is attributed to the fact that the human visual system is mostly sensitive to luminance changes in that portion of the spectrum. The perceived intensity increase monotonically for this scale.

Radiance default color scale (DEF): This is the default false coloring scale used in the Radiance global illumination software. ${ }^{1}$ It was designed to maximize the number of named colors while still depicting a progression from cold to hot.

Linearized optimal color scale (LOCS): LOCS is designed to create a maximum number of just noticeable differences (JNDs) while preserving a natural order. ${ }^{15}$ This scale is perceptually linearized (numerical color differences correspond to perceived color differences) and monotonically increasing in perceived intensity.

RBS color scale was selected because it is commonly used for visualization, although it also has a bad reputation. ${ }^{16}$ HBS was selected as another commonly used scale, in which the perceived intensity values increase monotonically across the scale. LOCS was selected because it is both monotonic and perceptually linear. Finally, DEF was selected as it is the default color scale in a commonly used light simulation program, Radiance. ${ }^{1}$ The color palettes of these four color scales can be found in the Appendix.

\section{EVALUATION}

In this paper, we performed both objective and subjective evaluations using 100 face images from the publicly available FERET face recognition dataset. ${ }^{4}$ Figure 2 presents example images from the dataset. Objective evaluation relied on three face recognition algorithms implemented in the evaluation framework by Korshunov et al. ${ }^{2}$ In subjective evaluation, we employed 50 subjects in online study.

\subsection{Objective Evaluation}

For objective evaluation, each face image was false colored using the combinations of the three point transformations functions plus a no-transformation condition and four color scales resulting in total 16 visualizations per face. The resulted
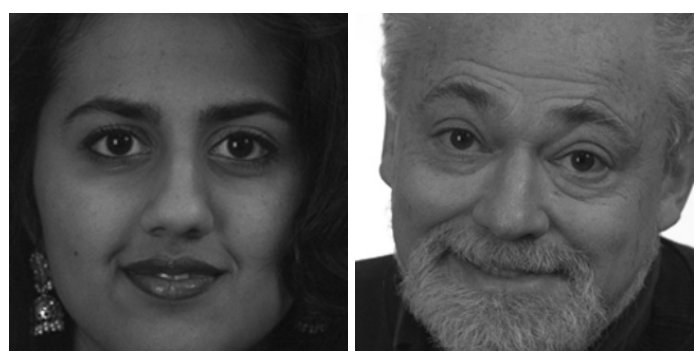

Figure 2: Sample images from FERET dataset. 



Figure 3: False colored image results for the two sample images.

false colored sample images are presented in Figure 3. To evaluate the recognizability of these false color visualizations, we used objective evaluation framework, ${ }^{2}$ which utilizes three face recognition algorithms implemented in OpenCV ${ }^{\ddagger}$ The face recognition algorithms are the principal component analysis (PCA) referred to as 'Eigen' ${ }^{17}$ linear discriminant analysis (LDA) referred to as 'Fisher', ${ }^{18}$ and local binary patterns referred to as 'LBPH' algorithm. ${ }^{19}$

\footnotetext{
${ }^{\ddagger} \mathrm{http} / / /$ opencv.willowgarage.com/wiki/
} 


\subsubsection{Results}

The visual inspection of Figure 3, showing different false colored images, reveal that not all compression type/color palette combination are equally effective. For example, the faces in the images obtained by using the HBS and LOCS remain mostly recognizable. This can be attributed to the fact that these color scales have a monotonic perceived intensity variation. However, the RBS and the DEF appear to apply strong enough distortions to faces, making them hard to recognize.

These visual observations are supported by the quantitative results that are obtained by using the above mentioned objective framework. Table 1 reports the face recognition accuracy obtained by running three different face recognition algorithms on all false colored images from the dataset. In this table, the lower accuracy numbers indicate a higher degree of privacy protection against face recognition algorithms. It can be seen that, on average, the 'LBPH' method is the most successful in recognizing false colored face images. However, for HIS-DEF combination, even 'LBPH' recognition shows a low accuracy of 0.11 , which means that out of 100 face images in the dataset, only 11 were correctly recognized by the algorithm. In general, the DEF color scale is the most effective in privacy protection, since it reduces the accuracy rates irrespective of the applied point transformation. Following DEF, RBS color scale is found to be the second most effective. The other two color scales, HBS and LOCS, are both ineffective against recognition algorithms, leading to high accuracy ratings.

Similar trends can be observed for 'Eigen' and 'Fisher' face recognition algorithms. However, the accuracy ratings of both of these algorithms are generally lower than the LBPH-based face recognition algorithm. For both algorithms LOG-RBS, SIG-DEF, and NOP-DEF methods yield a 0 accuracy value.

Based on the results in Table 1, it can be argued that the color scale is the critical factor that strongly influences the face recognition accuracy for the tested face recognition algorithms. It shows that a point transformation applied prior to the color mapping has little affect on the accuracy of the algorithms and, hence, has little contribution to privacy protection.

We also compare the obtained accuracy ratings with two methods from the literature, namely blurring and warping (Table 2). The lowest accuracy for these two methods is obtained for a blur kernel size of 55, which leads to accuracy value 0.14 of 'LBPH' recognition and significantly higher for other recognition methods. However, blurring is not only computationally more expensive than the proposed false coloring algorithm, but it is also irreversible when such a large kernel size of 55 is applied. False colored images can be reversed to obtain the images that are very close to originals (see the second rows for each sample face in Figure 3). The slight intensity differences between the originals and the reversed images mainly due to the compression functions.

\begin{tabular}{|c|c|c|c|c|c|c|c|c|c|c|c|c|}
\hline \multirow{2}{*}{$\begin{array}{ll}\text { Compression } & \text { Palette }\end{array}$} & \multicolumn{4}{|c|}{ LBPH } & \multicolumn{4}{|c|}{ Eigen } & \multicolumn{4}{|c|}{ Fisher } \\
\hline & RBS & DEF & HBS & LOCS & RBS & $\overline{\text { DEF }}$ & HBS & LOCS & $\overline{\text { RBS }}$ & $\overline{\text { DEF }}$ & HBS & LOCS \\
\hline LOG & 0.27 & 0.14 & 0.75 & 0.76 & 0.00 & 0.01 & 0.44 & 0.49 & 0.00 & 0.01 & 0.44 & 0.49 \\
\hline SIG & 0.41 & 0.14 & 0.78 & 0.50 & 0.01 & $\overline{0.00}$ & 0.61 & 0.46 & 0.01 & 0.00 & 0.61 & 0.46 \\
\hline HIS & 0.19 & 0.11 & 0.87 & 0.69 & 0.05 & 0.04 & 0.59 & 0.67 & 0.05 & 0.04 & 0.58 & 0.66 \\
\hline NOP & 0.46 & 0.13 & 0.76 & 0.44 & 0.01 & 0.00 & 0.60 & 0.37 & 0.01 & $\begin{array}{c}0.00 \\
\end{array}$ & 0.59 & 0.36 \\
\hline
\end{tabular}

Table 1: Face recognition accuracy rates results for false colored faces. The lower the value, the better the performance.

\begin{tabular}{|c|c|c|c|c|c|c|}
\hline & \multicolumn{2}{|c|}{ LBPH } & \multicolumn{2}{c|}{ Eigen } & \multicolumn{2}{c|}{ Fisher } \\
\hline \multirow{2}{*}{ Warping } & Strength level = 3 & Strength level =13 & Strength level =3 & Strength level = 13 & Strength level = 3 & Strength level = 13 \\
\cline { 2 - 7 } & 0.64 & 0.90 & 0.68 & 0.89 & 0.68 & 0.89 \\
\hline \multirow{2}{*}{ Blurring } & Kernel size = 5 & Kernel size = 55 & Kernel size =5 & Kernel size = 55 & Kernel size = 5 & Kernel size = 55 \\
\cline { 2 - 7 } & 0.72 & $\mathbf{0 . 1 4}$ & 0.89 & 0.79 & 0.89 & 0.79 \\
\hline
\end{tabular}

Table 2: Face recognition accuracy rates for warping and blurring. The lower the value, the better the performance.

\subsection{Subjective Evaluation}

A good privacy protection algorithm should provide effective protection not only against machines but also against human observers. We conducted online-based subjective experiments to evaluate this aspect of the proposed privacy protection method. For subjective evaluation, we used 10 faces from the FERET100 dataset. In our experimental design, we used a similar approach to that proposed by Rogowitz and Kalvin. ${ }^{3}$ In our web-based implementation, we asked participants to rate the recognizability of the false color visualizations with respect to the original image. The participants could see the 

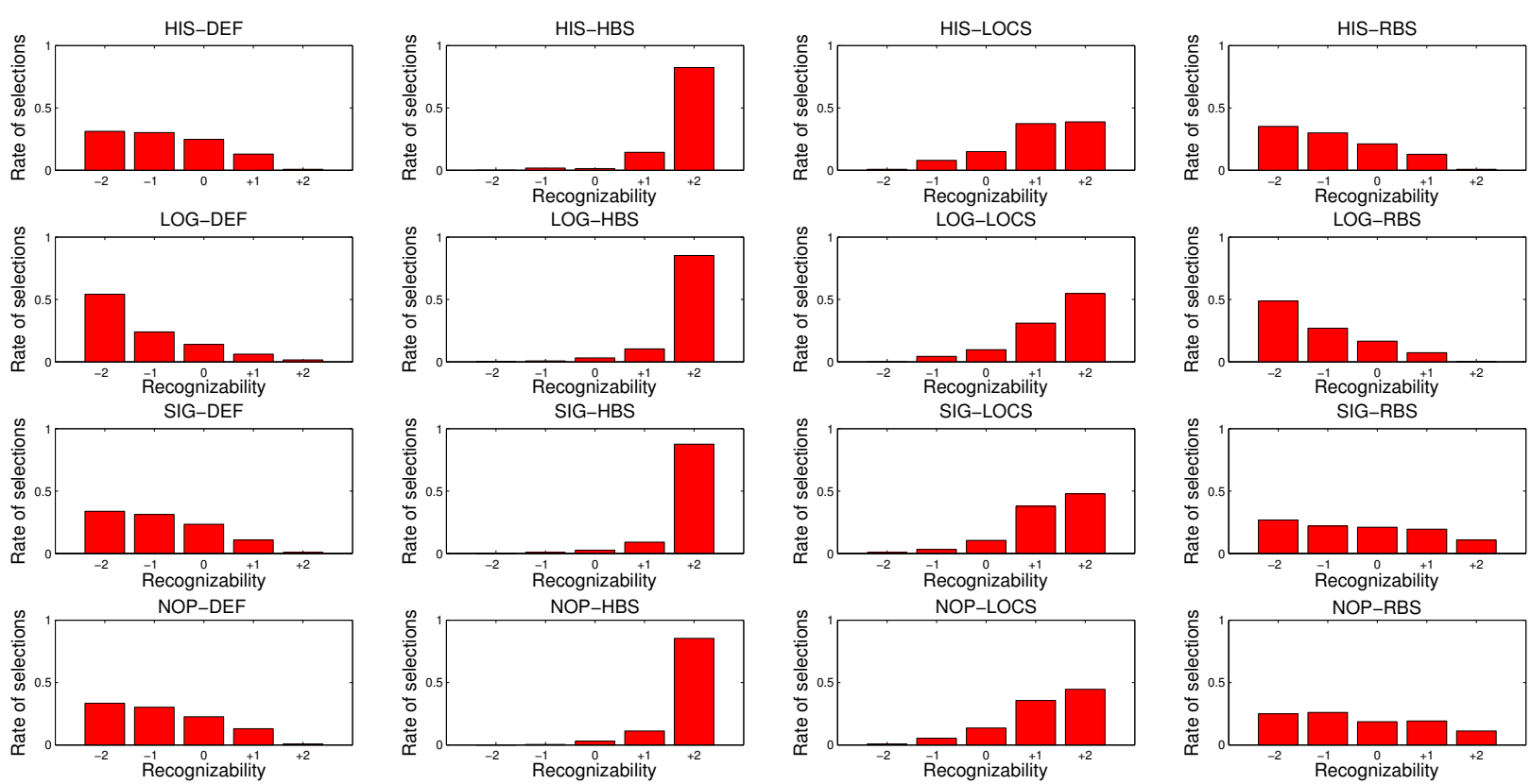

Figure 4: Cumulative results of the subjective experiments. The $\mathrm{x}$-axis represents the recognizability values and the $y$-axis represents participants' normalized selection counts.

original image on the left and the false color images in random order appearing as a 4 by 4 grid on the right. Under each false color image there was a drop-down list with the following options:

+2: Very likely to be recognizable

+1: Likely to be recognizable

0: May or may not be recognizable

-1: Unlikely to be recognizable

-2: Very unlikely to be recognizable

The experiment was comprised of 10 sessions, whereby a different input face image was used in each session. Each session ended when a participant indicated his or her responses for all of the 16 visualizations. The order of the sessions and the visualizations in each session were randomized to avoid any order-specific bias. The experiment had a web interface and therefore each participant took the experiment using his or her own computer system. The duration of a single experiment was approximately $15-20$ minutes. A total of 50 naïve subjects (36 males and 14 females) completed all sessions in full and their results were analyzed.

\subsubsection{Results}

The cumulative results of the subjective experiment are depicted in Figure 4. Each histogram in this figure indicates the responses for a single visualization method aggregated over all participants and face images. The y-axis is normalized to indicate the rate of selections. As can be seen from this figure, both DEF and RBS scales gave rise to right-skewed distributions whereas HBS and LOCS produced left-skewed ones. This suggests that the former two color scales are found less likely to be recognizable whereas the latter two are more likely to be recognizable. Similar to the results of objective evaluation, for all color scales, the compression/expansion methods do not seem to have a significant influence.

In order to obtain a global score for all methods, responses with values $-2,-1,+1$, and +2 were added together to obtain one single value. Therefore, a global score with large negative value means that the corresponding method produces less recognizable faces. The global scores computed for all false color protection methods are shown in Table 3, where 


\begin{tabular}{|c|cccc|}
\hline & DEF & RBS & LOCS & HBS \\
\hline LOG & $-\mathbf{3 5 2}$ & $-\mathbf{3 4 1}$ & 407 & 472 \\
SIG & -267 & -93 & 408 & 479 \\
HIS & -238 & -258 & 337 & 475 \\
NOP & -249 & -103 & 370 & 481 \\
\hline
\end{tabular}

Table 3: The global scores for each method. More negative scores indicate less recognizable methods. For example, LOG-DEF was rated as -2 or -1352 times out of a total of 500 ratings.

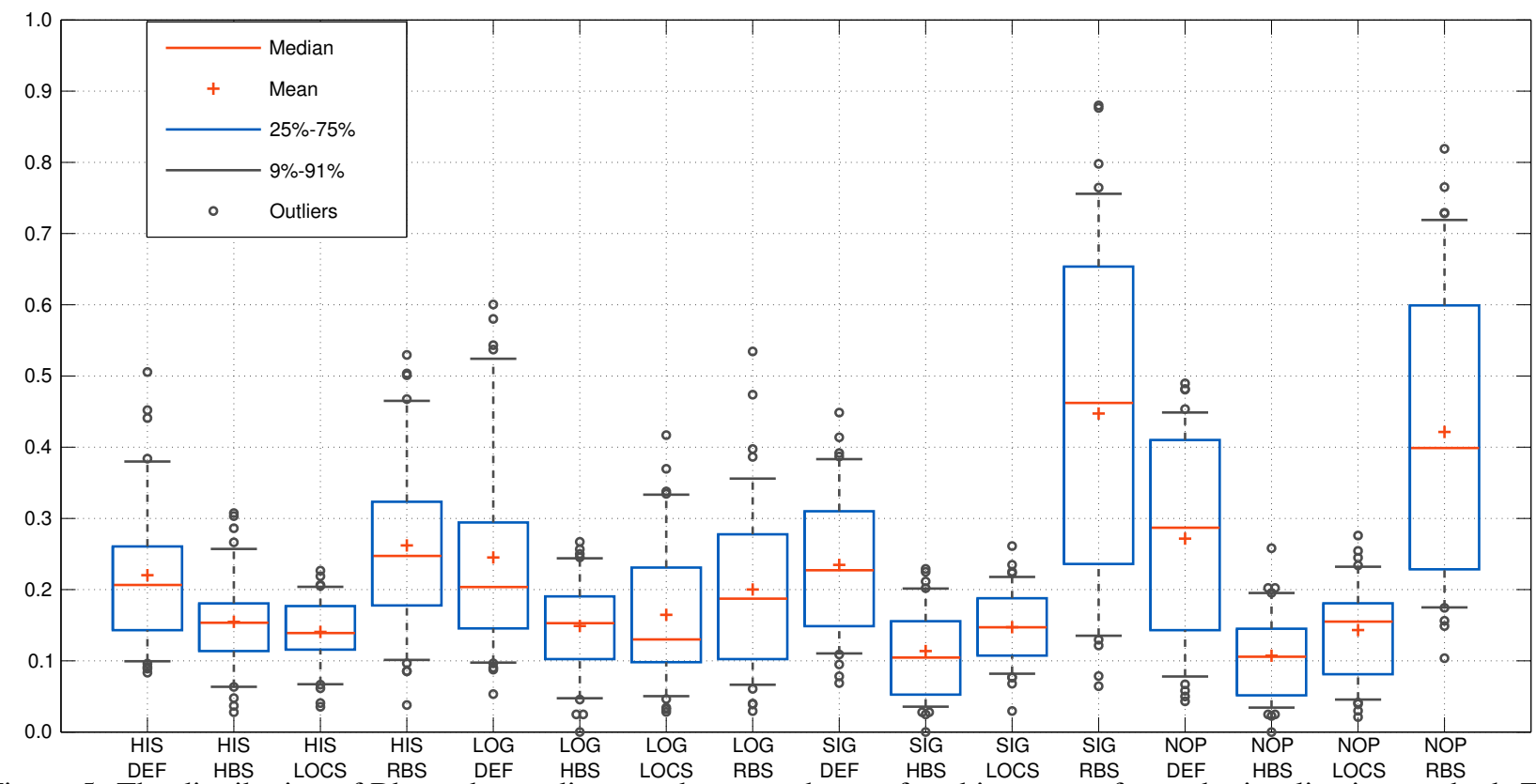

Figure 5: The distribution of Bhattacharya distances between the per-face histograms for each visualization method. The smaller the values of a distribution are the more consistent the corresponding method is for different faces.

the two largest negative scores are highlighted in bold. The table shows that LOG-DEF and LOG-RBS scales produce the least recognizable faces, while LOCS and HBS color scales lead to the easiest recognizable faces irrespective of the prior compression transformation.

To understand whether a given method's preferences vary across faces, we conducted further analysis of the subjective results. We computed the Bhattacharya distances between pairs of histograms that are obtained for different faces. For 10 faces used in the subjective study, we have $C(10,2)=45$ pairs, resulting in 45 different distance values for each of 16 methods. These distances are plotted in Figure 5, where different false color methods are shown in $\mathrm{x}$-axis and and Bhattacharya distances in $\mathrm{y}$-axis. In this figure, the smaller the values of a distribution, the more consistent the corresponding method is for different faces. However, a method can be consistently recognizable or consistently unrecognizable. The two most unrecognizable methods, similarly to objective evaluations, are LOG-DEF and LOG-RBS, with the latter varying slightly less across different faces. Hence, we conclude that LOG-RBS is the winner method in the subjective experiments with LOG-DEF being the close second.

The conclusion that the LOG-RBS method was found to produce the least recognizable images may be explained as follows. The logarithmic compression clumps together different intensity values more so than the other compression methods. Furthermore, the yellow colors dominate the RBS color space which has a masking effect over other hues due to the higher sensitivity of the human visual system to yellow. Finally, yellow has the smallest number of saturation steps which make it difficult to distinguish small saturation variations. ${ }^{20}$ These properties of LOG-RBS may have resulted in certain facial features to be lost when a face is visualized using this method, rendering it less recognizable.

We also investigated the correlation between the rankings of the user study and the rankings obtained by using the face recognition algorithms. For this purpose, we computed Spearman's rank correlation coefficient, ${ }^{21}$ which is a commonly 


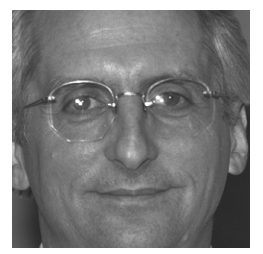

0.0

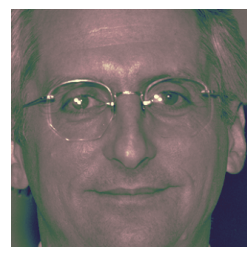

0.2

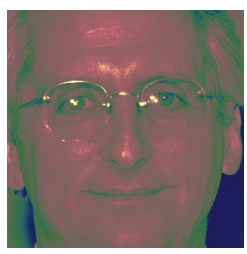

0.4

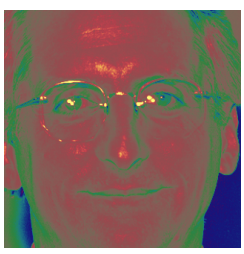

0.6

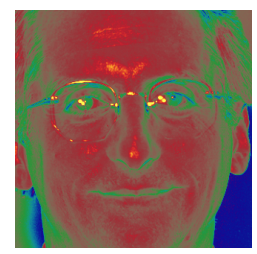

0.8

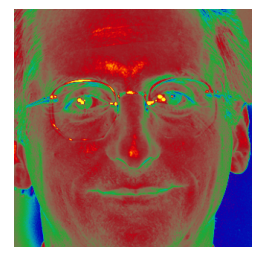

1.0

Figure 6: Interpolation between an original image and its false color visualization. The numbers indicate the weights given to the false color result. LOG-DEF is used as the visualization method.

used measure to compare ranked variables. We obtained a correlation value of $\rho=0.8645$ with LBPH and $\rho=0.8195$ with 'Eigen' and 'Fisher' algorithms. Such high correlation values indicate that false colored faces are perceived similarly by both human observers and face recognition algorithms, which is an important finding, since for more typical distortions (blurring or pixelization) the perception by humans and computers is different. ${ }^{22}$

Finally, we show the amount of privacy protection by using false colors is adjustable as the final result can be computed by interpolating the false color image with the original with different degrees of interpolation. For some applications where intelligibility is more important than privacy, a lower weight can be given to the false colored result to produce more intelligible images as shown in Figure 6.

\section{CONCLUSION AND FUTURE WORK}

Privacy protection in video surveillance is an important problem, and it will become even more important, as video surveillance is gains in popularity. However, simple methods for protecting privacy are not sufficient as they do not contain all the desired attributes that is expected from a good privacy protection algorithm. Privacy protected videos must be reversible if the need arises to view them as unprotected (e.g., during a criminal investigation). Furthermore, protected videos should not prohibit non-private statistics to be extracted. Also, the protected content should not be visually disturbing as it is the case with some of the existing privacy protection methods, such as scrambling and warping. Perhaps most importantly, the protection must be continuous: that is faces even in a single frame of a video should not remain unprotected. The algorithms that rely on computer vision techniques may therefore be vulnerable to this problem: if an algorithm fails to detect a sensitive region, it will remain unprotected.

This paper proposed false color based method, which aims to achieve the balance between the above mentioned desired attributes. The method is reversible since the compression and color scale tables can be inverted and is secure because these tables can be encrypted using a private key. The false colored representations do not prohibit collecting non-private information (for instance one can still count the number of people in an area without knowing their identities). And since the method does not rely on computer vision, it therefore is not affected by potential failures of detection or tracking algorithms.

The objective and subjective evaluations show that DEF and RBS color scales are the most suitable for privacy protection for both use cases, when face recognition algorithms and human subjects are the main observers of the protected images. Also, compression/expansion schemes demonstrate a significantly less effect on the strength of privacy protection compared to the color scales.

Several future research directions are possible. Firstly, the proposed algorithm can be evaluated using crowdsourcing. This would involve input from a large number of participants from very different backgrounds. The experimental task can be varied: instead of directly asking the degree of recognizability, one can design a task that indirectly evaluates this attribute. For example, one can ask whether a person whose images was previously shown appears in a given video clip. Such a design is likely to represent a more realistic surveillance scenario. Finally, the design of other compression algorithms and color palettes that are customized for protecting privacy can be an studied.

\section{ACKNOWLEDGMENTS}

This work was conducted in the framework of the EC funded Network of Excellence VideoSense. We are grateful to Okan Tarhan Tursun for his help with the experimental framework and all the anonymous participants who took our experiment. 


\section{REFERENCES}

[1] Larson, G. and Shakespeare, R., [Rendering with Radiance: The Art and Science of Lighting Visualization ], Computer Graphics and Geometric Modeling Series, Morgan Kaufmann (1998).

[2] Korshunov, P., Melle, A., Dugelay, J.-L., and Ebrahimi, T., "A framework for objective evaluation of privacy filters in video surveillance," in [SPIE Applications of Digital Image Processing XXXVI], 8856, Spie-Int Soc Optical Engineering, San Diego, California, USA (Aug. 2013).

[3] Rogowitz, B. and Kalvin, A. D., "The "which blair project": a quick visual method for evaluating perceptual color maps," in [Visualization, 2001. VIS'01. Proceedings], 183-556, IEEE (2001).

[4] Phillips, J. P., Moon, H., Rizvi, S. A., and Rauss, P. J., "The FERET Evaluation Methodology for Face-Recognition Algorithms," IEEE Transactions on Pattern Analysis and Machine Intelligence 22(10), 1090-1104 (2000).

[5] Korshunov, P. and Ebrahimi, T., "Using Warping for Privacy Protection in Video Surveillance," in [18th International Conference on Digital Signal Processing (DSP)], 1-6 (2013).

[6] Korshunov, P. and Ebrahimi, T., "Using Face Morphing to Protect Privacy," in [IEEE International Conference on Advanced Video and Signal-Based Surveillance (AVSS)], 208 - 213 (Aug. 2013).

[7] Benson, P. J., "Morph transformation of the facial image," Image and Vision Computing 12(10), 691 - 696 (1994).

[8] Dufaux, F. and Ebrahimi, T., "Scrambling for Privacy Protection in Video Surveillance Systems," IEEE Trans. on Circuits and Systems for Video Technology vol. 18(no. 8), 1168-1174 (2008).

[9] Cheung, S.-C., Venkatesh, M., Paruchuri, J., Zhao, J., and Nguyen, T., "Protecting and managing privacy information in video surveillance systems," in [Protecting Privacy in Video Surveillance], 11-33, Springer (2009).

[10] Martin, K. and Plataniotis, K. N., "Privacy protected surveillance using secure visual object coding," Circuits and Systems for Video Technology, IEEE Transactions on 18(8), 1152-1162 (2008).

[11] Erdélyi, A., Barát, T., Valet, P., Winkler, T., and Rinner, B., "Adaptive cartooning for privacy protection in camera networks," in [Proceedings of the International Conference on Advanced Video and Signal Based Surveillance ], 6 (2014).

[12] Akyüz, A. O., "False color visualization for hdr images," in [HDRi2013 - First International Conference and SME Workshop on HDR imaging ], (April 2013).

[13] Reinhard, E., Stark, M., Shirley, P., and Ferwerda, J., "Photographic tone reproduction for digital images," ACM Transactions on Graphics 21(3), 267-276 (2002).

[14] Gonzalez, R. C. and Woods, R. E., [Digital Image Processing], Addison-Wesley Longman Publishing Co., Inc., Boston, MA, USA, 2nd ed. (1992).

[15] Levkowitz, H. and Herman, G. T., "Color scales for image data," IEEE Comput. Graph. Appl. 12, $72-80$ (Jan. 1992).

[16] Rogowitz, B. and Treinish, L. A., "Data visualization: the end of the rainbow," Spectrum, IEEE 35(12), 52-59 (1998).

[17] Turk, M. A. and Pentland, A. P., "Face recognition using eigenfaces," in [Computer Vision and Pattern Recognition, 1991. Proceedings CVPR 91., IEEE Computer Society Conference on ], 586-591, IEEE (June 1991).

[18] Belhumeur, P. N., Hespanha, J. a. P., and Kriegman, D. J., "Eigenfaces vs. fisherfaces: Recognition using class specific linear projection," IEEE Trans. Pattern Anal. Mach. Intell. 19, 711-720 (July 1997).

[19] Ahonen, T., Hadid, A., and Pietikainen, M., "Face description with local binary patterns: Application to face recognition," IEEE Trans. Pattern Anal. Mach. Intell. 28, 2037-2041 (Dec. 2006).

[20] Wang, L., Giesen, J., McDonnell, K. T., Zolliker, P., and Mueller, K., "Color design for illustrative visualization," Visualization and Computer Graphics, IEEE Transactions on 14(6), 1739-1754 (2008).

[21] Myers, J. L., Well, A., and Lorch, R. F., [Research design and statistical analysis], Routledge (2010).

[22] Korshunov, P. and Ooi, W. T., "Video quality for face detection, recognition, and tracking," ACM Trans. Multimedia Comput. Commun. Appl. 7, 14:1-14:21 (Sept. 2011). 


\section{APPENDIX: COLOR PALETTES}

\begin{tabular}{|c|c|c|c|c|c|c|c|c|c|c|}
\hline & & & & & & & & & & \\
\hline & $\mathbf{R}$ & $\mathbf{G}$ & $\mathbf{B}$ & & $\mathbf{R}$ & $\mathbf{G}$ & $\begin{array}{l}\mathbf{B} \\
\end{array}$ & & $\mathbf{R}$ & $\mathbf{G}$ \\
\hline 0 & 111 & 8 & 132 & 86 & 18 & 174 & 81 & 172 & 159 & 34 \\
\hline 1 & 108 & 7 & 133 & 87 & 20 & 174 & 77 & 173 & 159 & 33 \\
\hline 2 & 105 & 7 & 134 & 88 & 21 & 173 & 73 & 174 & 160 & 32 \\
\hline 3 & 102 & 6 & 136 & 89 & 22 & 172 & 69 & 175 & 160 & 31 \\
\hline 4 & 98 & 6 & 137 & 90 & 24 & 172 & 66 & 176 & 161 & 29 \\
\hline 5 & 93 & 5 & 139 & 91 & 26 & 171 & 63 & 177 & 161 & 28 \\
\hline 6 & 89 & 4 & 141 & 92 & 28 & 170 & 60 & 178 & 162 & 27 \\
\hline 7 & 84 & 3 & 143 & 93 & 30 & 169 & 58 & 179 & 162 & 26 \\
\hline 8 & 79 & 2 & 145 & 94 & 32 & 168 & 57 & 180 & 163 & 25 \\
\hline 9 & 74 & 1 & 148 & 95 & 34 & 167 & 56 & 181 & 163 & 24 \\
\hline 10 & 68 & 0 & 150 & 96 & 37 & 166 & 55 & 182 & 164 & 23 \\
\hline 11 & 63 & 0 & 153 & 97 & 40 & 165 & 54 & 183 & 165 & 22 \\
\hline 12 & 57 & 0 & 155 & 98 & 42 & 164 & 54 & 184 & 165 & 21 \\
\hline 13 & 52 & 0 & 157 & 99 & 45 & 163 & 54 & 185 & 168 & 18 \\
\hline 14 & 46 & 0 & 160 & 100 & 48 & 162 & 55 & 186 & 170 & 16 \\
\hline 15 & 41 & 0 & 162 & 101 & 52 & 160 & 55 & 187 & 172 & 13 \\
\hline 16 & 36 & 0 & 164 & 102 & 55 & 158 & 56 & 188 & 175 & 11 \\
\hline 17 & 31 & 0 & 166 & 103 & 58 & 157 & 57 & 189 & 177 & 9 \\
\hline 18 & 26 & 0 & 168 & 104 & 62 & 155 & 57 & 190 & 180 & 7 \\
\hline 19 & 22 & 0 & 170 & 105 & 66 & 153 & 59 & 191 & 183 & 5 \\
\hline 20 & 18 & 0 & 172 & 106 & 69 & 152 & 60 & 192 & 185 & 3 \\
\hline 21 & 14 & 2 & 174 & 107 & 73 & 150 & 61 & 193 & 188 & 2 \\
\hline 22 & 11 & 4 & 175 & 108 & 77 & 148 & 63 & 194 & 191 & 1 \\
\hline 23 & 8 & 7 & 176 & 109 & 81 & 146 & 64 & 195 & 194 & 0 \\
\hline 24 & 7 & 9 & 177 & 110 & 84 & 144 & 66 & 196 & 197 & 0 \\
\hline 25 & 6 & 11 & 177 & 111 & 88 & 142 & 67 & 197 & 199 & 0 \\
\hline 26 & 5 & 13 & 178 & 112 & 92 & 139 & 69 & 198 & 202 & 0 \\
\hline 27 & 4 & 16 & 178 & 113 & 96 & 137 & 70 & 199 & 205 & 0 \\
\hline 28 & 3 & 18 & 179 & 114 & 99 & 135 & 72 & 200 & 207 & 0 \\
\hline 29 & 2 & 21 & 180 & 115 & 103 & 133 & 73 & 201 & 210 & 2 \\
\hline 30 & 1 & 24 & 180 & 116 & 107 & 131 & 75 & 202 & 213 & 3 \\
\hline 31 & 1 & 28 & 181 & 117 & 110 & 128 & 76 & 203 & 215 & 6 \\
\hline 32 & 0 & 31 & 181 & 118 & 113 & 126 & 77 & 204 & 217 & 8 \\
\hline 33 & 0 & 35 & 182 & 119 & 117 & 124 & 78 & 205 & 219 & 11 \\
\hline 34 & 0 & 38 & 182 & 120 & 120 & 121 & 79 & 206 & 220 & 13 \\
\hline 35 & 0 & 42 & 183 & 121 & 123 & 119 & 80 & 207 & 222 & 17 \\
\hline 36 & 0 & 46 & 184 & 122 & 126 & 117 & 80 & 208 & 224 & 20 \\
\hline 37 & 0 & 50 & 184 & 123 & 128 & 114 & 81 & 209 & 226 & 24 \\
\hline 38 & 0 & 54 & 184 & 124 & 131 & 112 & 81 & 210 & 227 & 28 \\
\hline 39 & 0 & 58 & 185 & 125 & 133 & 110 & 81 & 211 & 229 & 32 \\
\hline 40 & 0 & 63 & 185 & 126 & 135 & 108 & 80 & 212 & 231 & 37 \\
\hline 41 & 0 & 67 & 186 & 127 & 136 & 106 & 80 & 213 & 232 & 42 \\
\hline 42 & 0 & 71 & 186 & 128 & 137 & 105 & 80 & 214 & 234 & 47 \\
\hline 43 & 0 & 76 & 186 & 129 & 138 & 104 & 79 & 215 & 236 & 52 \\
\hline 44 & 0 & 80 & 187 & 130 & 139 & 102 & 79 & 216 & 237 & 57 \\
\hline 45 & 0 & 84 & 187 & 131 & 140 & 101 & 79 & 217 & 239 & 63 \\
\hline 46 & 0 & 89 & 187 & 132 & 141 & 100 & 78 & 218 & 240 & 68 \\
\hline 47 & 0 & 93 & 187 & 133 & 142 & 98 & 78 & 219 & 242 & 74 \\
\hline 48 & 1 & 97 & 187 & 134 & 143 & 96 & 77 & 220 & 243 & 79 \\
\hline 49 & 1 & 102 & 187 & 135 & 144 & 95 & 76 & 221 & 245 & 85 \\
\hline $\mathbf{5 0}$ & 1 & 106 & 187 & 136 & 144 & 93 & 76 & 222 & 246 & 91 \\
\hline 51 & 2 & 110 & 187 & 137 & 145 & 92 & 75 & 223 & 247 & 96 \\
\hline 52 & 2 & 114 & 187 & 138 & 146 & 90 & 74 & 224 & 248 & 102 \\
\hline 53 & 3 & 118 & 186 & 139 & 146 & 89 & 73 & 225 & 250 & 108 \\
\hline 54 & 3 & 122 & 186 & 140 & 147 & 87 & 73 & 226 & 251 & 113 \\
\hline 55 & 4 & 126 & 186 & 141 & 148 & 85 & 72 & 227 & 252 & 118 \\
\hline 56 & 4 & 130 & 185 & 142 & 148 & 84 & 71 & 228 & 253 & 123 \\
\hline 57 & 4 & 133 & 185 & 143 & 149 & 82 & 70 & 229 & 254 & 128 \\
\hline 58 & 5 & 137 & 184 & 144 & 149 & 80 & 69 & 230 & 254 & 133 \\
\hline 59 & 5 & 140 & 183 & 145 & 150 & 79 & 68 & 231 & 255 & 138 \\
\hline 60 & 6 & 143 & 182 & 146 & 150 & 77 & 67 & 232 & 255 & 143 \\
\hline 61 & 6 & 146 & 181 & 147 & 151 & 75 & 66 & 233 & 255 & 148 \\
\hline 62 & 6 & 149 & 180 & 148 & 151 & 73 & 65 & 234 & 255 & 154 \\
\hline 63 & 7 & 151 & 179 & 149 & 151 & 72 & 64 & 235 & 255 & 159 \\
\hline 64 & 7 & 154 & 178 & 150 & 152 & 70 & 63 & 236 & 255 & 165 \\
\hline 65 & 7 & 156 & 177 & 151 & 152 & 68 & 62 & 237 & 255 & 170 \\
\hline 66 & 8 & 158 & 175 & 152 & 153 & 66 & 61 & 238 & 255 & 176 \\
\hline 67 & 8 & 161 & 172 & 153 & 153 & 65 & 60 & 239 & 255 & 181 \\
\hline 68 & 9 & 163 & 169 & 154 & 153 & 63 & 59 & 240 & 255 & 187 \\
\hline 69 & 9 & 165 & 165 & 155 & 154 & 61 & 58 & 241 & 255 & 192 \\
\hline 70 & 9 & 167 & 161 & 156 & 154 & 60 & 57 & 242 & 255 & 198 \\
\hline 71 & 9 & 169 & 157 & 157 & 154 & 58 & 56 & 243 & 255 & 203 \\
\hline 72 & 10 & 170 & 153 & 158 & 154 & 56 & 55 & 244 & 255 & 208 \\
\hline 73 & 10 & 172 & 148 & 159 & 155 & 55 & 54 & 245 & 255 & 213 \\
\hline 74 & 10 & 173 & 143 & 160 & 155 & 53 & 53 & 246 & 255 & 218 \\
\hline 75 & 11 & 174 & 138 & 161 & 155 & 51 & 51 & 247 & 255 & 223 \\
\hline 76 & 11 & 174 & 133 & 162 & 156 & 50 & 50 & 248 & 255 & 227 \\
\hline 77 & 11 & 175 & 127 & 163 & 156 & 48 & 49 & 249 & 255 & 232 \\
\hline 78 & 12 & 175 & 122 & 164 & 156 & 46 & 48 & 250 & 255 & 236 \\
\hline 79 & 12 & 176 & 117 & 165 & 157 & 45 & 47 & 251 & 254 & 240 \\
\hline 80 & 13 & 176 & 111 & 166 & 157 & 43 & 46 & 252 & 254 & 243 \\
\hline 81 & 14 & 176 & 106 & 167 & 157 & 42 & 45 & 253 & 254 & 246 \\
\hline 82 & 14 & 176 & 101 & 168 & 158 & 40 & 44 & 254 & 254 & 249 \\
\hline 83 & 15 & 175 & 95 & 169 & 158 & 39 & 43 & 255 & 254 & 252 \\
\hline 84 & 16 & 175 & 90 & 170 & 158 & 37 & 42 & & & \\
\hline 85 & 17 & 175 & 86 & 171 & 159 & 36 & 41 & & & \\
\hline
\end{tabular}

Table 4: Color palettes for DEF and HBS color maps. 


\begin{tabular}{|c|c|c|c|c|c|c|c|c|c|c|}
\hline & & & & & & & & & & \\
\hline & $\mathbf{R}$ & $\mathbf{G}$ & B & & $\mathbf{R}$ & $\mathbf{G}$ & $\bar{B}$ & & $\mathbf{R}$ & $\bar{G}$ \\
\hline 0 & 0 & 0 & 0 & 86 & 135 & 6 & 0 & 172 & 135 & 200 \\
\hline 1 & 0 & 0 & 0 & 87 & 135 & 8 & 0 & 173 & 135 & 203 \\
\hline 2 & 0 & 0 & 0 & 88 & 135 & 9 & 0 & 174 & 135 & 205 \\
\hline 3 & 1 & 0 & 0 & 89 & 135 & 10 & 0 & 175 & 135 & 210 \\
\hline 4 & 2 & 0 & 0 & 90 & 135 & 11 & 0 & 176 & 135 & 214 \\
\hline 5 & 2 & 0 & 0 & 91 & 135 & 13 & 0 & 177 & 135 & 218 \\
\hline 6 & 3 & 0 & 0 & 92 & 135 & 13 & 0 & 178 & 135 & 222 \\
\hline 7 & 3 & 0 & 0 & 93 & 135 & 15 & 0 & 179 & 135 & 226 \\
\hline 8 & 4 & 0 & 0 & 94 & 135 & 17 & 0 & 180 & 135 & 231 \\
\hline 9 & 5 & 0 & 0 & 95 & 135 & 17 & 0 & 181 & 135 & 236 \\
\hline 10 & 5 & 0 & 0 & 96 & 135 & 19 & 0 & 182 & 135 & 239 \\
\hline 11 & 6 & 0 & 0 & 97 & 135 & 21 & 0 & 183 & 135 & 244 \\
\hline 12 & 7 & 0 & 0 & 98 & 135 & 22 & 0 & 184 & 135 & 249 \\
\hline 13 & 7 & 0 & 0 & 99 & 135 & 23 & 0 & 185 & 135 & 254 \\
\hline 14 & 8 & 0 & 0 & 100 & 135 & 25 & 0 & 186 & 135 & 255 \\
\hline 15 & 9 & 0 & 0 & 101 & 135 & 26 & 0 & 187 & 135 & 255 \\
\hline 16 & 9 & 0 & 0 & 102 & 135 & 27 & 0 & 188 & 135 & 255 \\
\hline 17 & 10 & 0 & 0 & 103 & 135 & 29 & 0 & 189 & 135 & 255 \\
\hline 18 & 11 & 0 & 0 & 104 & 135 & 31 & 0 & 190 & 135 & 255 \\
\hline 19 & 12 & 0 & 0 & 105 & 135 & 32 & 0 & 191 & 135 & 255 \\
\hline 20 & 13 & 0 & 0 & 106 & 135 & 33 & 0 & 192 & 135 & 255 \\
\hline 21 & 14 & 0 & 0 & 107 & 135 & 35 & 0 & 193 & 135 & 255 \\
\hline 22 & 15 & 0 & 0 & 108 & 135 & 36 & 0 & 194 & 135 & 255 \\
\hline 23 & 16 & 0 & 0 & 109 & 135 & 38 & 0 & 195 & 135 & 255 \\
\hline 24 & 17 & 0 & 0 & 110 & 135 & 40 & 0 & 196 & 135 & 255 \\
\hline 25 & 18 & 0 & 0 & 111 & 135 & 42 & 0 & 197 & 135 & 255 \\
\hline 26 & 19 & 0 & 0 & 112 & 135 & 44 & 0 & 198 & 135 & 255 \\
\hline 27 & 20 & 0 & 0 & 113 & 135 & 46 & 0 & 199 & 135 & 255 \\
\hline 28 & 21 & 0 & 0 & 114 & 135 & 47 & 0 & 200 & 135 & 255 \\
\hline 29 & 22 & 0 & 0 & 115 & 135 & 49 & 0 & 201 & 135 & 255 \\
\hline 30 & 23 & 0 & 0 & 116 & 135 & 51 & 0 & 202 & 135 & 255 \\
\hline 31 & 25 & 0 & 0 & 117 & 135 & 52 & 0 & 203 & 135 & 255 \\
\hline 32 & 26 & 0 & 0 & 118 & 135 & 54 & 0 & 204 & 135 & 255 \\
\hline 33 & 27 & 0 & 0 & 119 & 135 & 56 & 0 & 205 & 135 & 255 \\
\hline 34 & 28 & 0 & 0 & 120 & 135 & 57 & 0 & 206 & 135 & 255 \\
\hline 35 & 30 & 0 & 0 & 121 & 135 & 59 & 0 & 207 & 135 & 255 \\
\hline 36 & 31 & 0 & 0 & 122 & 135 & 62 & 0 & 208 & 135 & 255 \\
\hline 37 & 33 & 0 & 0 & 123 & 135 & 63 & 0 & 209 & 135 & 255 \\
\hline 38 & 34 & 0 & 0 & 124 & 135 & 65 & 0 & 210 & 135 & 255 \\
\hline 39 & 35 & 0 & 0 & 125 & 135 & 67 & 0 & 211 & 135 & 255 \\
\hline 40 & 37 & 0 & 0 & 126 & 135 & 69 & 0 & 212 & 135 & 255 \\
\hline 41 & 39 & 0 & 0 & 127 & 135 & 72 & 0 & 213 & 135 & 255 \\
\hline 42 & 40 & 0 & 0 & 128 & 135 & 73 & 0 & 214 & 135 & 255 \\
\hline 43 & 43 & 0 & 0 & 129 & 135 & 76 & 0 & 215 & 135 & 255 \\
\hline 44 & 45 & 0 & 0 & 130 & 135 & 78 & 0 & 216 & 135 & 255 \\
\hline 45 & 46 & 0 & 0 & 131 & 135 & 80 & 0 & 217 & 135 & 255 \\
\hline 46 & 49 & 0 & 0 & 132 & 135 & 82 & 0 & 218 & 135 & 255 \\
\hline 47 & 51 & 0 & 0 & 133 & 135 & 84 & 0 & 219 & 135 & 255 \\
\hline 48 & 53 & 0 & 0 & 134 & 135 & 87 & 0 & 220 & 135 & 255 \\
\hline 49 & 54 & 0 & 0 & 135 & 135 & 88 & 0 & 221 & 135 & 255 \\
\hline 50 & 56 & 0 & 0 & 136 & 135 & 90 & 0 & 222 & 135 & 255 \\
\hline 51 & 58 & 0 & 0 & 137 & 135 & 93 & 0 & 223 & 135 & 255 \\
\hline 52 & 60 & 0 & 0 & 138 & 135 & 95 & 0 & 224 & 135 & 255 \\
\hline 53 & 62 & 0 & 0 & 139 & 135 & 98 & 0 & 225 & 135 & 255 \\
\hline 54 & 64 & 0 & 0 & 140 & 135 & 101 & 0 & 226 & 135 & 255 \\
\hline 55 & $\begin{array}{l}07 \\
67\end{array}$ & 0 & 0 & 141 & 135 & 103 & 0 & 227 & 135 & 255 \\
\hline 56 & 69 & 0 & 0 & 142 & 135 & 106 & 0 & 228 & 135 & 255 \\
\hline 57 & 71 & 0 & 0 & 143 & 135 & $\begin{array}{l}107\end{array}$ & 0 & 229 & 135 & 255 \\
\hline 58 & 74 & 0 & 0 & 144 & 135 & 110 & 0 & 230 & 135 & 255 \\
\hline 59 & 76 & 0 & 0 & 145 & 135 & 113 & 0 & 231 & 135 & 255 \\
\hline 60 & 80 & 0 & 0 & 146 & 135 & 115 & 0 & 232 & 135 & 255 \\
\hline 61 & 81 & 0 & 0 & 147 & 135 & 118 & 0 & 233 & 135 & 255 \\
\hline 62 & 84 & 0 & 0 & 148 & 135 & $\begin{array}{l}121 \\
\end{array}$ & 0 & 234 & 140 & 255 \\
\hline 63 & 86 & 0 & 0 & 149 & 135 & 124 & 0 & 235 & 146 & 255 \\
\hline 64 & 89 & 0 & 0 & 150 & 135 & 127 & 0 & 236 & 153 & 255 \\
\hline 65 & 92 & 0 & 0 & 151 & 135 & 129 & 0 & 237 & 156 & 255 \\
\hline 66 & 94 & 0 & 0 & 152 & 135 & 133 & 0 & 238 & 161 & 255 \\
\hline 67 & 97 & 0 & 0 & 153 & 135 & 135 & 0 & 239 & 168 & 255 \\
\hline 68 & 100 & 0 & 0 & 154 & 135 & 138 & 0 & 240 & 172 & 255 \\
\hline 69 & 103 & 0 & 0 & 155 & 135 & 141 & 0 & 241 & 177 & 255 \\
\hline 70 & 106 & 0 & 0 & 156 & 135 & 144 & 0 & 242 & 182 & 255 \\
\hline 71 & 109 & 0 & 0 & 157 & 135 & 148 & 0 & 243 & 189 & 255 \\
\hline $\begin{array}{l}1 \\
72\end{array}$ & 112 & 0 & 0 & 158 & $\begin{array}{l}135\end{array}$ & $\begin{array}{l}140 \\
150\end{array}$ & 0 & 244 & $\begin{array}{l}192 \\
1\end{array}$ & 255 \\
\hline 73 & 115 & 0 & 0 & 159 & 135 & 155 & 0 & 245 & 199 & 255 \\
\hline 74 & 117 & 0 & 0 & 160 & 135 & 157 & 0 & 246 & 204 & 255 \\
\hline 75 & 122 & 0 & 0 & 161 & 135 & 160 & 0 & 247 & 210 & 255 \\
\hline 76 & 126 & 0 & 0 & 162 & 135 & 163 & 0 & 248 & 215 & 255 \\
\hline 77 & 128 & 0 & 0 & 163 & 135 & 166 & 0 & 249 & 220 & 255 \\
\hline 78 & 131 & 0 & 0 & 164 & 135 & 170 & 0 & 250 & 225 & 255 \\
\hline 79 & 135 & 0 & 0 & 165 & 135 & 174 & 0 & 251 & 232 & 255 \\
\hline 80 & 135 & 0 & 0 & 166 & 135 & 177 & 0 & 252 & 236 & 255 \\
\hline 81 & 135 & 1 & 0 & 167 & 135 & 180 & 0 & 253 & 240 & 255 \\
\hline $\begin{array}{l}81 \\
82\end{array}$ & 135 & 2 & 0 & 168 & 135 & 184 & 0 & 254 & 248 & 255 \\
\hline 83 & 135 & 3 & 0 & 169 & 135 & 188 & 0 & 255 & 255 & 255 \\
\hline $\begin{array}{l}85 \\
84\end{array}$ & $\begin{array}{l}135 \\
\end{array}$ & 4 & 0 & 170 & 135 & $\begin{array}{l}192 \\
192\end{array}$ & 0 & & & \\
\hline 85 & 135 & 6 & 0 & 171 & 135 & 195 & 0 & & & \\
\hline
\end{tabular}

Table 5: Color palettes for LOCS and RBS color maps. 